Images et réalité de l'huile d'olive pour le consommateur européen

Oléagineux, Corps Gras, Lipides. Volume 11, Numéro 3, 199-202, MAI-JUIN 2004, Les évolutions

Auteur(s) : Guillaume DILAS

FNCG 118 avenue Peretti 92200 Neuilly Tél. : 0146372560 ; Fax : 0146371560

\title{
ARTICLE
}

Suite à la campagne européenne menée entre 2000 et 2002 pour la promotion de l'huile d'olive (la septième), la direction générale de l'Agriculture de la Commission a commandité une étude d'évaluation de cette action. Comme il se doit, le but était en particulier d'apprécier dans quelle mesure les objectifs de la campagne - consolidation de la place de l'huile d'olive dans les pays producteurs (Grèce, Italie, Portugal et Espagne) et développement de la consommation dans les pays non-producteurs - avaient été atteints. Etablie à partir d'un questionnaire type de quarante questions, renseigné par sondage téléphonique, une enquête a été ainsi conduite auprès d'un panel de consommateurs d'environ 1000 personnes par pays (UE à 15), auquel a été adjoint dans un second volet un panel de médecins généralistes. Pour l'Espagne et le Danemark cette évaluation a également concerné les responsables de grandes surfaces et des importateurs.Publiée sous le titre "The evaluation of the 7th European campaign (2000-2002) to promote the consumption of olive oil in the European Union ", l'étude est aujourd'hui disponible sur le site de la direction générale de l'Agriculture de la Commission européenne ${ }^{\underline{1}}$.De ce document réunissant un ensemble assez considérable de données (qui ne sont pas pour certaines sans poser des problèmes d'interprétation : voir en particulier les graphiques concernant l'évolution des réponses entre 1996 et 2002) et de tableaux, il nous a semblé possible d'extraire de manière non exhaustive (et non officielle) quelques éléments permettant de fixer certains des traits différentiels de l'huile d'olive pour le consommateur européen.Le "coût", l'image de "produit santé ", la fréquence d'utilisation comparée à celle d'autres corps gras (huiles végétales, beurre, margarine), sa notoriété, sont les aspects que nous avons essayé de cerner, ceci à travers les réponses des panels de six pays : deux pays producteurs (I'Espagne, l'Italie), trois pays présentant un fort potentiel de consommation (France, Allemagne, Royaume-Uni), et un pays du Nord (Suède).

\section{Notoriété différentielle}

En réponse à la question : "Quel type de corps gras connaissez-vous, ne serait-ce que de nom ? " seule la première réponse donnée spontanément étant retenue, l'huile d'olive apparaît de loin comme le corps gras qui bénéficie de la plus grande notoriété dans les 6 pays choisis (tableau 1)( Tableau 1 ). Sans grande surprise, cette notoriété frôle les $80 \%$ dans les pays producteurs. Et mis à part le Royaume-Uni sa notoriété dépasse à elle seule celle de l'ensemble des autres huiles végétales. 
En Suède, elle est même citée plus souvent qu'en France $(45,7 \%$ contre $38,6 \%)$, pays pourtant producteur et où la consommation a connu une forte progression depuis dix ans.

La notoriété des différents corps gras montre par ailleurs une certaine homogénéité entre les consommateurs. Ainsi l'huile de tournesol arrive en seconde position partout sauf en Suède. Pour ce dernier la notoriété des margarines y est même supérieure à celle de l'ensemble des huiles végétales $(15,8 \%$ contre $13,5 \%)$.

Tableau 1 Question : Quel type d'huile et de matière grasse connaissez-vous, ne serait-ce que de nom ? (Première réponse citée spontanément).

\begin{tabular}{|c|c|c|c|c|c|c|c|}
\hline \multicolumn{2}{|l|}{ En \% } & Espagne & Italie & France & Allemagne & $\begin{array}{l}\text { Royaume- } \\
\text { Uni }\end{array}$ & Suède \\
\hline \multicolumn{2}{|l|}{ Huile d'olive } & 79,2 & 79,4 & 38,6 & 27,3 & 29,7 & 45,7 \\
\hline \multicolumn{2}{|c|}{ Autres huiles végétales dont, notamment } & 11,3 & 10,4 & 34,4 & 25,0 & 43,9 & 13,5 \\
\hline & $\begin{array}{ll}\text { Huile } & \text { de } \\
\text { tournesol } & \end{array}$ & 9,7 & 2,9 & 18,2 & 16,6 & 22,1 & 1,3 \\
\hline Huile de colza & 0,1 & 0,0 & 1,2 & 2,4 & 0,6 & 5,7 & \\
\hline Huile d'arachide & 0,0 & 1,0 & 9,0 & 0,4 & 0,1 & 0,0 & \\
\hline $\begin{array}{l}\text { Huile végétale (en tant que } \\
\text { telle) }\end{array}$ & 1,2 & 2,3 & 3,5 & 4,4 & 18,1 & 3,2 & \\
\hline \multicolumn{2}{|l|}{ Margarine } & 0,0 & 0,3 & 4,5 & 7,7 & 2,5 & 15,8 \\
\hline \multicolumn{2}{|l|}{ Beurre } & 1,0 & 1,5 & 11,5 & 10,1 & 2,2 & 8,2 \\
\hline \multicolumn{2}{|l|}{ Graisse animale } & 0,1 & 0,1 & 0,4 & 1,8 & 5,8 & 0,3 \\
\hline \multicolumn{2}{|l|}{ Autres corps gras } & 6,6 & 5,7 & 7,2 & 16,7 & 5,2 & 13,3 \\
\hline \multirow{2}{*}{\multicolumn{2}{|c|}{ Ne sait pas }} & 1,7 & 2,6 & 3,4 & 11,4 & 10,7 & 3,2 \\
\hline & & 100,0 & 100,0 & 100,0 & 100,0 & 100,0 & 100,0 \\
\hline
\end{tabular}

\section{Fréquence d'utilisation}

Quelle est la place de l'huile d'olive dans l'alimentation des européens en termes de fréquence d'utilisation comparée à celle des autres corps gras?

Utilisée au minimum une à deux fois par semaine (tableau 2), I'huile d'olive confirme sa bonne notoriété. On peut dégager ici deux grandes tendances: d'une part une consommation de type 
quotidienne (Italie $90 \%$, Espagne $85 \%$ et France $37 \%$ ) et d'autre part une consommation de type hebdomadaire (au moins une fois) en Allemagne et au Royaume Uni. Il est à noter qu'en Suède 60 \% des personnes interrogées utilisent l'huile d'olive au moins une fois par semaine.

Si I'huile d'olive fait désormais partie des mœurs alimentaires des Européens, les tableaux précisant les fréquences de consommation des autres corps gras permettent de mieux circonscrire sa place.

Ainsi l'étude avec les tableaux 3, 4 et 5 met en évidence trois types de consommation quotidienne de corps gras :

- - l'Italie et l'Espagne sont les plus gros consommateurs quotidiens d'huile d'olive ;

- - le beurre est le corps gras le plus consommé au quotidien en France et en Allemagne (plus de $50 \%$ des sondés l'utilisent tous les jours);

- - le Royaume-Uni et la Suède affichent quant à eux leur préférence pour la margarine mais dans des proportions moins marquées (respectivement 42 et $35 \%$ ).

Cette segmentation est à nuancer car mis à part l'Italie et l'Espagne, où la consommation quotidienne de corps gras est presque exclusivement tournée vers l'huile d'olive, les ressortissants des autres pays consomment quotidiennement plusieurs corps gras. En France, outre le beurre et I'huile d'olive, les autres huiles végétales sont également utilisées le plus souvent au quotidien.

La Suède fait définitivement cas à part : la consommation quotidienne de corps gras y est beaucoup moins marquée par la prédominance d'un seul produit. En effet, si $35 \%$ des Suédois interrogés consomment quotidiennement de la margarine, ils sont $25 \%$ à consommer du beurre et $21 \%$ de I'huile d'olive.

Il convient de noter que l'ensemble de ces données doit être analysé en fonction de l'usage des corps gras (assaisonnement, cuisson, friture, tartine) qui n'apparaissent pas dans cette étude ; en effet les différents corps gras ne sont pas « interchangeables » en toutes circonstances.

S'il apparaît que l'huile d'olive a largement conquis le quotidien des ménages en Europe, toutefois environ un quart des consommateurs allemands et suédois avouent ne jamais en consommer ou alors de manière occasionnelle. Pour le Royaume-Uni c'est un peu plus du tiers des sondés qui se range dans cette catégorie avec notamment $23 \%$ déclarant ne l'avoir jamais utilisée.

Tableau 2 Fréquence d'utilisation de l'huile d'olive (\%).

\begin{tabular}{|l||l|l|l|l|l|l|}
\hline En \% & Espagne & Italie & France & Allemagne & Royaume-Uni & Suède \\
\hline Tous les jours & 85,8 & 90,5 & 37,5 & 17,1 & 12,9 & 21,2 \\
\hline Plus de 2 fois par semaine & 6,1 & 5,6 & 20,8 & 22,0 & 16,9 & 20,1 \\
\hline 1 à 2 fois par semaine & 2,2 & 1,1 & 17,6 & 25,0 & 22,7 & 21,1 \\
\hline Chaque mois & 0,5 & 0,5 & 6,1 & 10,2 & 10,4 & 10,6 \\
\hline
\end{tabular}




\begin{tabular}{|l||l|l|l|l|l|l|}
\hline Occasionnellement & 1,5 & 0,5 & 7,8 & 13,0 & 13,8 & 13,2 \\
\hline Jamais & 2,2 & 1,5 & 9,8 & 10,8 & 23,0 & 13,6 \\
\hline Ne sait pas & 1,7 & 0,3 & 0,4 & 1,9 & 0,3 & 0,2 \\
\hline & 100,0 & 100,0 & 100,0 & 100,0 & 100,0 & 100,0 \\
\hline \hline
\end{tabular}

Tableau 3 Fréquence d'utilisation des autres huiles végétales (tout type de graines ou fruits).

\begin{tabular}{|l|l|l|l|l|l|l|}
\hline En \% & Espagne & Italie & France & Allemagne & Royaume-Uni & Suède \\
\hline Tous les jours & 17,0 & 6,9 & 28,0 & 17,3 & 7,6 & 12,4 \\
\hline Plus de 2 fois par semaine & 10,6 & 10,4 & 20,1 & 16,4 & 13,7 & 14,7 \\
\hline 1 à 2 fois par semaine & 16,5 & 23,5 & 22,3 & 26,1 & 27,7 & 21,2 \\
\hline Chaque mois & 6,0 & 13,4 & 7,4 & 12,1 & 11,5 & 15,0 \\
\hline Occasionnellement & 13,2 & 14,0 & 9,4 & 12,7 & 12,8 & 18,1 \\
\hline Jamais & 30,2 & 24,2 & 11,4 & 12,4 & 24,3 & 17,5 \\
\hline Ne sait pas & 6,5 & 7,6 & 1,4 & 3,0 & 2,4 & 1,1 \\
\hline & 100,0 & 100,0 & 100,0 & 100,0 & 100,0 & 100,0 \\
\hline
\end{tabular}

Tableau 4 Fréquence d'utilisation du beurre.

\begin{tabular}{|l||l|l|l|l|l|l|}
\hline En \% & Espagne & Italie & France & Allemagne & Royaume-Uni & Suède \\
\hline Tous les jours & 8,1 & 7,1 & 52,1 & 53,5 & 32,8 & 25,1 \\
\hline Plus de 2 fois par semaine & 8,1 & 14,8 & 9,6 & 7,5 & 7,3 & 8,9 \\
\hline 1 à 2 fois par semaine & 11,9 & 25,2 & 15,6 & 10,9 & 9,7 & 10,8 \\
\hline Chaque mois & 6,9 & 14,7 & 3,1 & 6,4 & 6,4 & 12,3 \\
\hline Occasionnellement & 16,1 & 15,3 & 4,5 & 10,0 & 7,1 & 15,9 \\
\hline Jamais & 36,8 & 10,8 & 7,3 & 9,9 & 30,6 & 25,7 \\
\hline Ne sait pas & 12,1 & 12,1 & 7,8 & 1,8 & 6,1 & 1,3 \\
\hline \hline & 100,0 & 100,0 & 100,0 & 100,0 & 100,0 & 100,0 \\
\hline
\end{tabular}


Tableau 5 Fréquence d'utilisation de la margarine.

\begin{tabular}{|l||l|l|l|l|l|l|}
\hline En \% & Espagne & Italie & France & Allemagne & Royaume-Uni & Suède \\
\hline Tous les jours & 4,3 & 0,8 & 21,7 & 40,5 & 42,0 & 35,5 \\
\hline Plus de 2 fois par semaine & 6,9 & 2,8 & 8,1 & 10,6 & 7,5 & 20,9 \\
\hline 1 à 2 fois par semaine & 12,1 & 9,3 & 15,1 & 15,8 & 8,6 & 16,9 \\
\hline Chaque mois & 5,3 & 10,1 & 4,4 & 8,1 & 7,7 & 9,4 \\
\hline Occasionnellement & 13,2 & 18,4 & 6,6 & 13,0 & 5,0 & 6,8 \\
\hline Jamais & 41,1 & 39,2 & 35,9 & 11,1 & 26,0 & 9,2 \\
\hline Ne sait pas & 17,1 & 19,4 & 8,2 & 1,0 & 3,2 & 1,3 \\
\hline \hline
\end{tabular}

L'huile d'olive : usage à chaud, usage à froid

L'huile d'olive s'utilise-t-elle à chaud ? à froid ? (tableaux 6 et 7). Les réponses concernant l'usage à froid n'apportent pas de grands enseignements. L'huile d'olive est largement associée à un usage à froid : l'assaisonnement.

Concernant l'usage à chaud, on constate que la grande majorité des personnes interrogées quel que soit le pays sont " tout à fait d'accord " ou " plutôt d'accord " sur ce type d'utilisation. Les chiffres varient ainsi de $55 \%$ en Suède à plus de $90 \%$ en Espagne, et laissent ainsi supposer au moins pour les pays non-producteurs des « possibilités » d'utilisation importantes.

Les chiffres les plus élevés exprimant une réticence (" plutôt pas d'accord " voire " totalement pas d'accord ") à associer l'huile d'olive à un usage à chaud varient de $26 \%$ (Allemagne) à $17 \%$ (Suède). En France ils sont de $21 \%$.

Tableau 6 Question posée : I'huile d'olive convient pour les plats froids ?

\begin{tabular}{|l|l|l|l|l|l|l|}
\hline En \% & Espagne & Italie & France & Allemagne & Royaume-Uni & Suède \\
\hline Tout à fait d'accord & 69,8 & 66,4 & 54,3 & 47,7 & 44,5 & 39,4 \\
\hline Plutôt d'accord & 23,1 & 26,2 & 24,3 & 30,3 & 27,1 & 22,7 \\
\hline Ni d'accord ni pas d'accord & 4,6 & 2,2 & 5,2 & 4,7 & 11,7 & 20,2 \\
\hline Plutôt pas d'accord & 0,3 & 2,6 & 9,1 & 8,4 & 6,8 & 6,0 \\
\hline
\end{tabular}




\begin{tabular}{|l|l|l|l|l|l|l|}
\hline Totalement pas d'accord & 0,9 & 1,8 & 5,1 & 3,4 & 5,7 & 9,2 \\
\hline \hline Ne sait pas & 1,3 & 0,8 & 2,0 & 5,4 & 4,3 & 2,4 \\
\hline \hline & 100,0 & 100,0 & 100,0 & 100,0 & 100,0 & 100,0 \\
\hline
\end{tabular}

Tableau 7 Question posée : I'huile d'olive convient pour les plats chauds ?

\begin{tabular}{|l|l|l|l|l|l|l|}
\hline En \% & Espagne & Italie & France & Allemagne & Royaume-Uni & Suède \\
\hline \hline Tout à fait d'accord & 67,7 & 46,0 & 38,3 & 30,3 & 51,0 & 32,4 \\
\hline Plutôt d'accord & 23,7 & 33,8 & 30,8 & 30,7 & 25,8 & 23,5 \\
\hline \hline Ni d'accord ni pas d'accord & 4,6 & 5,7 & 7,1 & 6,5 & 10,4 & 24,5 \\
\hline Plutôt pas d'accord & 1,7 & 9,0 & 12,5 & 19,1 & 6,4 & 8,4 \\
\hline Totalement pas d'accord & 1,1 & 3,6 & 9,3 & 7,5 & 3,4 & 8,6 \\
\hline \hline Ne sait pas & 1,2 & 2,0 & 2,0 & 6,0 & 3.1 & 2,5 \\
\hline \hline & 100,0 & 100,0 & 100,0 & 100,0 & 100,0 & 100,0 \\
\hline \hline
\end{tabular}

\section{L’huile d'olive, produit santé}

Incontestablement l'huile d'olive a acquis une image de " produit santé » (tableau 8)( Tableau 8 ). En effet la tendance est la même partout bien qu'elle reste plus ou moins profondément marquée selon les pays.

Ainsi, d'une manière globale, environ $75 \%$ des Espagnols et des Français interrogés sont " plutôt d'accord" voire "tout à fait d'accord" pour considérer que l'huile d'olive peut prévenir et combattre les maladies cardio-vasculaires. Cette proportion passe à $60 \%$ en Allemagne, à environ $55 \%$ au Royaume-Uni et en Suède contre $50 \%$ en Italie.

Le chiffre le plus remarquable concerne l'Italie où environ un tiers des personnes interrogées n'ont pas d'avis sur la question. Faut-il voir ici l'effet d'une tradition de consommation se préoccupant peu d'une information (et d'une légitimation) scientifique?

Enfin, c'est en Suède, en Allemagne et au Royaume-Uni que l'on trouve les taux d'opinions opposées à l'idée que l'huile d'olive peut prévenir et combattre les maladies cardio-vasculaires les plus élevés, mais dans de faibles proportions (entre 10 et $15 \%$ ). 
Tableau 8 Question posée : pensez-vous que l'huile d'olive peut prévenir et combattre les maladies cardiovasculaires?

\begin{tabular}{|l|l|l|l|l|l|l|}
\hline En \% & Espagne & Italie & France & Allemagne & Royaume-Uni & Suède \\
\hline Tout à fait d'accord & 46,2 & 25,0 & 41,6 & 30,3 & 26,5 & 27,0 \\
\hline Plutôt d'accord & 30,2 & 26,6 & 33,8 & 31,4 & 31,8 & 26,4 \\
\hline Ni d'accord ni pas d'accord & 9,7 & 6,6 & 10,2 & 10,8 & 25,5 & 27,8 \\
\hline Plutôt pas d'accord & 1,1 & 5,0 & 4,9 & 10,3 & 6,1 & 6,0 \\
\hline Totalement pas d'accord & 1,4 & 3,0 & 2,8 & 2,7 & 3,6 & 7,9 \\
\hline \hline Ne sait pas & 11,4 & 33,8 & 6,7 & 14,5 & 6,4 & 4,9 \\
\hline \hline
\end{tabular}

\section{L'huile d'olive, produit cher}

D'une manière générale, l'huile d'olive est considérée comme un produit onéreux dans l'ensemble des pays. Toutefois la lecture de ce tableau ne doit pas occulter le fait que le consommateur européen ne paye pas le même prix pour une même huile d'olive partout en Europe (tableau 9).

C'est en France et en Espagne que les consommateurs sont les plus nombreux à juger l'huile d'olive chère avec, respectivement, $61 \%$ et $71 \%$ d'avis "tout à fait d'accord " ou " plutôt d'accord". Notons toutefois qu'au Royaume-Uni $30 \%$ des personnes interrogées sont "plutôt pas d'accord " voire "totalement pas d'accord" avec cette affirmation. C'est le pourcentage le plus élevé. Cette opinion est à rapprocher de celle de l'Allemagne.

Tableau 9 Question posée : I'huile d'olive est-elle un produit cher?

\begin{tabular}{|l|l|l|l|l|l|l|}
\hline En \% & Espagne & Italie & France & Allemagne & Royaume-Uni & Suède \\
\hline \hline Tout à fait d'accord & 30,8 & 16,4 & 35,5 & 25,6 & 25,7 & 20,8 \\
\hline \hline Plutôt d'accord & 31,1 & 39,0 & 36,1 & 27,6 & 24,5 & 24,4 \\
\hline Ni d'accord ni pas d'accord & 20,9 & 16,8 & 8,3 & 11,8 & 15,5 & 31,8 \\
\hline Plutôt pas d'accord & 8,1 & 14,8 & 11,8 & 19,3 & 21,0 & 9,4 \\
\hline Totalement pas d'accord & 4,6 & 8,5 & 5,5 & 7,9 & 8,8 & 10,4 \\
\hline \hline Ne sait pas & 4,5 & 4,5 & 2,9 & 8,0 & 4,5 & 3,2 \\
\hline \hline
\end{tabular}




\section{Quelle évolution?}

Avant de conclure, rappelons l'évolution de la consommation d'huile d'olive (consommation des ménages et utilisation par les IAA) (tableau 10). L'enquête de la Commission montre que I'huile d'olive peut être considérée comme un corps gras de référence aux yeux des Européens et les chiffres de consommation le confirment. Le marché de l'huile d'olive est en pleine évolution, reste à savoir jusqu'à quel point les courbes continueront de monter.

En France en particulier, cette " expansion » de l'huile d'olive en volume se produit dans un contexte général de baisse de la consommation directe des huiles végétales par les ménages - l'huile d'olive a ainsi dépassé en valeur l'huile de tournesol, et sa part de marché est passée de $9 \%$ en 1994 à $22 \%$ en 2003 contre 67 et 44,6\% pour l'huile de tournesol (consommation directe par les ménages ; source ONIOL). Au point que l'on peut se poser la question de savoir si cette évolution de sa consommation n'a pas déjà entraîné et entraînera dans l'avenir, un déplacement des comportements alimentaires traditionnels en matière de corps gras ? Cette question vaut en fait pour les trois principaux marchés en forte progression (France, Allemagne, Royaume-Uni) et appellerait naturellement une étude.

Tableau 10 Evolution des consommations en Europe.

\begin{tabular}{|l|l|l|}
\hline En milliers de tonnes & 1994/1995 & 2002/2003 (prov.) \\
\hline Espagne & 420,0 & 620,0 \\
\hline Italie & 675,0 & 750,0 \\
\hline France & 41,6 & 95,0 \\
\hline Allemagne & 16,9 & 38,9 \\
\hline Royaume-Uni & 17,3 & 34,0 \\
\hline Suède & 1,0 & 5,0 \\
\hline \hline
\end{tabular}

1 http://europa.eu.int/comm/agriculture/prom/eval/olive/index_en.htm. 